\title{
Experience of surgical morbidity after palliative surgery in patients with gastric carcinoma
}

\author{
Luis F. Oñate-Ocaña ${ }^{1}$, Guadalupe Méndez-Cruz ${ }^{1}$, Roberto Hernández-Ramos ${ }^{1}$, Mauricio Becker ${ }^{1}$, \\ José F. Carrillo ${ }^{2}$, Roberto Herrera-Goepfert ${ }^{3}$, Vincenzo Aiello-Crocifoglio ${ }^{1}$, Francisco Ochoa-Carrillo ${ }^{2}$, \\ and Arturo Beltrán-Ortega ${ }^{2}$ \\ ${ }^{1}$ Clinica de Neoplasias Gástricas, Departamento de Gastroenterología, Instituto Nacional de Cancerología, San Fernando 22, Colonia Sección \\ XVI, Tlalpan, D.F. 14080, Mexico City, Mexico \\ ${ }^{2}$ Clínica de Neoplasias Gástricas, Departamento de Gastroenterología, División de Cirugía, Instituto Nacional de Cancerología, Mexico City, \\ Mexico \\ ${ }^{3}$ Departamento de Patología, Instituto Nacional de Cancerología, Mexico City, Mexico
}

\begin{abstract}
Background. Indications for palliative surgery in gastric carcinoma (GC) are controversial. Our aim was to describe the results of palliative surgery in GC in terms of operative morbidity and survival.

Methods. We conducted a retrospective cohort study of patients with GC, who were divided into three groups: resection with microscopic residual disease (R1), palliative resection with macroscopic residual disease (R2), and gastrojejunostomy. Comparisons were tested with analysis of variance (ANOVA) or $\chi^{2}$ test, and the Kaplan-Meier method was used for survival analysis.

Results. One hundred and thirty-two patients were included in the study: 21 had R1, 71 had R2, and 40 had gastrojejunostomy. Surgical morbidity was recorded in 4 patients $(19 \%), 23$ patients $(32.4 \%)$, and 1 patient $(2.5 \%)$ in each of the three groups, respectively $(P=0.001)$. Operative mortality occurred in 6 patients $(8.5 \%)$ from the $R 2$ group and in $1(2.5 \%)$ patient from the gastrojejunostomy group $(P=0.406)$. Median survivals of the R1, R2, and gastrojejunostomy groups were $\mathbf{2 2 . 8}$ months (95\% confidence interval [CI], 16.4-29.3), 12.4 (95\% CI, 9.01-15.8) months, and 6.4 months (95\% CI, 0-14.6), respectively $(P=\mathbf{0 . 0 7 8})$

Conclusion. $\mathrm{R} 1$ resections and gastrojejunostomy were associated with low surgical morbidity and mortality, unlike R2 resection; in this group, surgical morbidity and mortality was high. Therefore, the benefit of palliative resection in the presence of extensive residual disease should be balanced against the risk of surgical morbidity.
\end{abstract}

Key words Gastric cancer · Metastasis · Gastrectomy · Palliative surgery $\cdot$ Gastrojejunostomy

Offprint requests to: L.F. Oñate-Ocaña

Received: March 1, 2007 / Accepted: October 2, 2007

\section{Introduction}

The incidence of gastric carcinoma (GC) has diminished over the past few decades. Notwithstanding this, it remains the most frequent cause of gastrointestinal cancer and the second highest cause of cancer-related mortality worldwide [1].

Radical gastrectomy is the treatment of choice for patients with GC $[1,2]$. Nevertheless, surgical resection with curative intent can be offered to only a small proportion of patients in Mexico because locally advanced disease or metastases are present at the time of diagnosis in two-thirds of patients [3]. In this important group, we can only attempt palliative management, including best medical support, chemotherapy, radiotherapy, or surgery [3, 4].

Palliative surgery is defined as a surgical procedure designed to alleviate symptoms and to prevent the appearance of complications $[4,5]$. In general terms, it is indicated for the management of pain, obstruction, bleeding, or perforation, and its objective is to improve the quality of life (QOL) of patients with GC [4-6]. However, in some circumstances, chemotherapy or radiotherapy can achieve adequate palliation in the absence of palliative surgery [7].

Palliative surgery does not seek to offer cure options, even when it can increase the lifespan in patients with locally advanced or metastatic illness [4]. Patients who can opt for a palliative surgical intervention are those with advanced locoregional or metastatic disease according to the malignant tumor (TNM) classification of the American Joint Committee on Cancer (AJCC) [8], and those who are not candidates for a resection with curative intent because of comorbidity or poor performance status.

Despite the importance and widespread use of these procedures, there is a paucity of information providing 
answers to many questions. We previously published a method for predicting surgical morbidity after gastrectomy for GC, but palliative surgery was not specifically considered [9].

Therefore, the objective of this study was to describe the impact of palliative surgery regarding operative morbidity, mortality, and survival in a cohort of patients with GC treated at a cancer center.

\section{Patients and methods}

\section{Patients}

This was a retrospective study of patients with GC treated at the Gastroenterology Department at the Instituto Nacional de Cancerología (INCan) Hospital in Mexico City from January 1987 to December 2005. Inclusion criteria comprised subjects of any age or sex who had had endoscopy and endoscopic biopsy of gastric adenocarcinoma and who were submitted to resection or gastrojejunostomy. Criteria for defining palliative resection included overt malignant activity found by the surgeon during laparotomy and considered as residual macroscopic neoplastic activity, defined as palliative resection with macroscopic residual disease (R2) [8]. The presence of microscopic activity in the surgical margin of the gastrectomy was not considered palliative resection, but was defined as resection with microscopic residual disease (R1). However, we included these patients as a control group to test differences in morbidity and survival.

Relevant clinical and radiological data were recorded, including clinical and pathological data for TNM classification, histopathological variables, treatment received, and surgical morbidity and mortality. The sixth edition of the AJCC's TNM staging system [8] was used to define clinical and pathological staging. The diagnosis of M1 disease was always based on the biopsy findings of metastatic lesions.

\section{Statistical analysis}

Patients were allocated to one of three groups according to type of surgery; R1, R2, and gastrojejunostomy. Factors associated with operative morbidity and mortality in these groups were studied by analysis of variance (ANOVA) and $\chi^{2}$ tests, for continuous and categorical variables, respectively. Survival analysis was performed by the Kaplan-Meier method [10], and the differences among groups were tested by the Log-rank test [11]. SPSS software for Windows version 10 was used to make all calculations (1999; SPSS, Chicago, IL, USA). A probability value of 0.05 or less was considered significant, and two-tailed statistics were considered in all cases.

\section{Results}

During the study period, 132 patients who met the inclusion criteria constituted the database for analysis. There were 62 women and 70 men (mean age, 54.4 years; range, 21-82 years; SD, 13.5). Twenty-one patients $(15.9 \%)$ underwent resection with microscopic residual disease (R1), 71 had palliative resection with macroscopic residual disease (R2), and 40 patients had gastrojejunostomy.

Major patient clinical and pathological variables in each of the allocated groups are listed in Table 1. The type of gastrectomy and lymph node dissection associated with each group and their surgical morbidities are shown in Table 2 . Surgical morbidity and mortality were higher in the R2 group than in the others. With respect to the R2 group, 4 of 39 patients $(10.3 \%)$ who underwent subtotal gastrectomy presented with anastomotic dehiscence, as opposed to $5(15.6 \%)$ of 32 patients who underwent total gastrectomy $(P=0.526)$.

Six patients $(28.6 \%)$ in the $\mathrm{R} 1$ group received adjuvant chemotherapy, while seven R2-group patients $(9.9 \%)$ and 10 gastrojejunostomy-group patients (25\%) received palliative chemotherapy. No patient in the R1 or gastrojejunostomy groups received radiotherapy, but eight R2-group patients (11.3\%) received palliative radiotherapy. No patient in this cohort received concomitant or sequential chemoradiation. Recurrences after resection in the $\mathrm{R} 1$ group were diagnosed in eight patients $(38 \%)$; six of them died of progressive disease during the study's follow-up period.

All patients in the $\mathrm{R} 2$ group presented with progression of cancer, and 26 of them died during the follow-up period of the study. Forty-five patients with extensive neoplastic activity and poor performance status were lost to follow-up.

Among the patients with gastrojejunostomy, all presented with progressive disease and 13 of them died during the study's follow-up period. Twenty-three patients with extensive neoplastic activity were lost to follow-up.

The median overall survival of the cohort was 15.3 months (95\% confidence interval [95\% CI], 8.3-22.4). Median overall survivals of the R1, R2, and gastrojejunostomy were 22.8 months (95\% CI, 16.4-29.3), 12.4 months (95\% CI, 9.01-15.8), and 6.4 months (95\% CI, $0-14.6)$, respectively $(P=0.078)$. Kaplan-Meier survival curves for these three groups are depicted in Fig. 1.

\section{Discussion}

More than $75 \%$ of our patients at the INCan Hospital present with advanced locoregional or metastatic disease, and surgical resection can be offered only to 
Table 1. Demographic and clinical data of the cohort

\begin{tabular}{|c|c|c|c|}
\hline & $\mathrm{R} 1(n=21)$ & $\mathrm{R} 2(n=71)$ & $\mathrm{Gj}(n=40)$ \\
\hline Age, years, mean (SD) & $55.9(11.8)$ & $55.4(15.2)$ & $58.4(11)$ \\
\hline $\operatorname{Sex}(F: M)$ & 11:10 & $35: 36$ & $16: 24$ \\
\hline Hemoglobin, g/dl, mean (SD) & $12.4(2.7)$ & $11.6(2.6)$ & $10.6(3.3)$ \\
\hline Hematocrit, \%, mean (SD) & $38.2(6.7)$ & $34.8(7.1)$ & $33.7(9.9)$ \\
\hline Albumin, g/dl, mean (SD) & $3.52(0.46)$ & $3.4(0.68)$ & $3.19(0.77)$ \\
\hline Lymphocytes, cells $/ \mathrm{mm}^{3}$, mean (SD) & $1819(747)$ & $1800(771)$ & $1460(638)$ \\
\hline \multicolumn{4}{|l|}{ Laurèn classification } \\
\hline Intestinal & 6 & 34 & 21 \\
\hline Diffuse & 12 & 29 & 18 \\
\hline Mixed & 3 & 8 & 1 \\
\hline \multicolumn{4}{|l|}{ Differentiation degree } \\
\hline Well & 0 & 6 & 5 \\
\hline Moderate & 6 & 19 & 10 \\
\hline Poor & 15 & 46 & 25 \\
\hline \multicolumn{4}{|l|}{ Location } \\
\hline Proximal & 5 & 14 & 0 \\
\hline Body & 1 & 5 & 2 \\
\hline Distal & 11 & 41 & 35 \\
\hline Pangastric & 4 & 11 & 3 \\
\hline \multicolumn{4}{|l|}{ Stage } \\
\hline II & 3 & 0 & 0 \\
\hline IIIa & 4 & $3^{\mathrm{a}}$ & $1^{\mathrm{b}}$ \\
\hline IIIb & 8 & 0 & 0 \\
\hline IV & 6 & 68 & 39 \\
\hline Ascites & 0 & 3 & 0 \\
\hline Palpable tumor & 5 & 15 & 2 \\
\hline Distant metastases & 4 & 41 & 24 \\
\hline
\end{tabular}

R1, Microscopic residual disease; R2, macroscopic residual disease; $\mathrm{Gj}$, gastrojejunostomy

${ }^{a}$ Three patients in the R2 group, classified as T4N0M0, were considered unfit for multiorgan resection, and gastrectomy was performed with macroscopic residual disease

${ }^{\mathrm{b}}$ One patient in the Gj group, of advanced age and with T4N0M0 disease, was unfit for multiorgan resection

Table 2. Types of surgery and operative morbidity and mortality

\begin{tabular}{|c|c|c|c|}
\hline & $\mathrm{R} 1$ & $\mathrm{R} 2$ & $\mathrm{Gj}$ \\
\hline \multicolumn{4}{|l|}{ Gastrectomy } \\
\hline Total & 9 & 32 & - \\
\hline Subtotal & 12 & 39 & - \\
\hline \multicolumn{4}{|l|}{ Lymphadenectomy } \\
\hline D0/D1 & 15 & 57 & - \\
\hline $\mathrm{D} 2 / \mathrm{D} 1+$ & 6 & 14 & - \\
\hline Operative morbidity* & $4(19 \%)$ & $23(32.4 \%)$ & $1(2.5 \%)$ \\
\hline Anastomotic dehiscence & - & 9 & - \\
\hline Bleeding & 1 & 2 & - \\
\hline Early dumping & - & 2 & - \\
\hline Pneumonia & - & 2 & - \\
\hline Myocardial infarction & - & 2 & - \\
\hline Lung atelectasis & 1 & 2 & - \\
\hline Abdominal abscess & 1 & 1 & - \\
\hline Intestinal occlusion & 1 & 1 & - \\
\hline Anastomotic stenosis & - & 1 & - \\
\hline Gastric fundus perforation & - & 1 & - \\
\hline Cerebral infarction & - & - & 1 \\
\hline Operative mortality** & 0 & $6(8.5 \%)$ & $1(2.5 \%)$ \\
\hline
\end{tabular}

$\mathrm{R} 1$, Microscopic residual disease; $\mathrm{R} 2$, macroscopic residual disease; $\mathrm{Gj}$, gastrojejunostomy $* P=0.001, * * P=0.406$ 


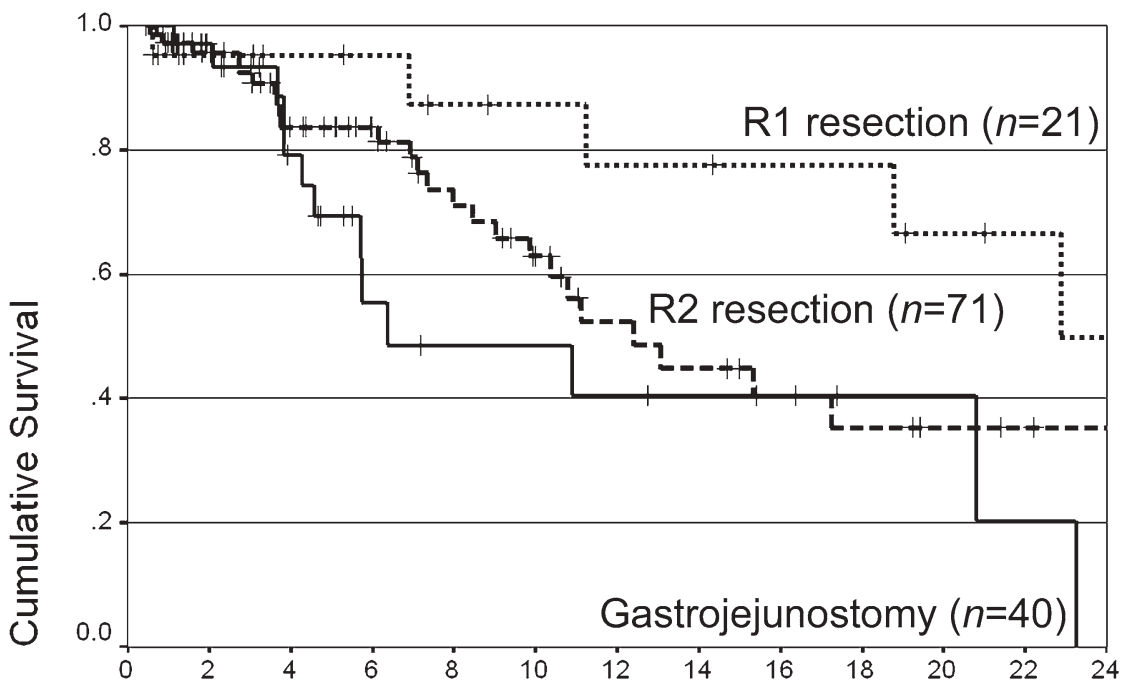

Time (months)

\begin{tabular}{|c|c|c|c|c|c|c|c|c|c|c|c|c|c|}
\hline \multicolumn{13}{|c|}{ Patients at risk } & \\
\hline R1 & 21 & 15 & 13 & 12 & 10 & 9 & 8 & 8 & 7 & 7 & 5 & & \\
\hline R2 & 71 & 61 & 46 & 36 & 27 & 20 & 14 & 12 & 9 & 7 & 5 & & \\
\hline Gj & 40 & 25 & 16 & 8 & 6 & 6 & 5 & 4 & 3 & 2 & 2 & 1 & \\
\hline
\end{tabular}

Fig. 1. Kaplan-Meier overall survival curves according to intent of the surgery. $R 1$ and $R 2$, microscopic and macroscopic residual disease, respectively; $G j$, gastrojejunostomy; + , censored cases. $P=0.078$
$33 \%$ of patients [3]. A substantial number of these patients receive palliative chemotherapy because it can improve quality of life by decreasing pain, increasing tolerance for meals, and allowing the patient to gain weight [7]. However, some patients experience complications that require palliative surgery as the best therapeutic option [4-6].

Palliative care has been defined by the World Health Organization (WHO) as "The total active care of patients whose disease is not responsive to curative treatment. Control of pain, of other symptoms, and of psychological, social, and spiritual problems is paramount. The goal of palliative care is the achievement of the best quality of life for patients and their families" [12].

In this study, we have reported the results of surgery in 132 Mexican patients with GC. We included R1 resections because the inclusion of such a group provided a control group to show differences in morbidity and survival.

The R2 group is particularly important. Patients had disease of a more advanced stage than those in the other groups analyzed, and they had low immunonutritional status (Table 1). Probably, that is the reason why surgical morbidity and mortality were significantly higher in the $\mathrm{R} 2$ group than in the other two groups.
The main weakness of the present study is the retrospective nature of the information. However, there is a paucity of information regarding this problem in Mexico and in other Latin American countries. As well, the follow-up was poor. Most patients in the three groups were lost to follow-up, because the majority were referred to local clinics in the country for end-of-life medical care.

In the past, subtotal gastrectomy was considered a safe and useful procedure for the palliation of distal GC. However, total gastrectomy was considered a dangerous operation for palliation in patients with proximal or pangastric disease location [13, 14]. However, surgical trends and postoperative care have changed for stage IV GC.

In a recent study, the survival of 105 patients with stage IV disease was reported in terms of the palliativeresection event: 81 of these patients (77.1\%) did not undergo resection but did receive palliative chemotherapy (some received chemoradiation), and 24 (22.9\%) underwent palliative gastric resection. The mean survival times were 5.9 months (95\% CI, 4.2-7.6) and 16.3 months (95\% CI, 4.3-28.8), respectively [15].

These data suggest that palliative resection combined with adjuvant therapy may improve survival in a selected group of fit patients with stage IV gastric cancer. Our suggestion is that palliative gastrectomy plus chemora- 
diation should be compared to systemic palliative chemotherapy or chemoradiation in a randomized clinical trial.

It is very important for the surgeon to select the most suitable patients for palliative resection in this setting, with respect to effective palliation and a good expectancy of surviving the operation, because case selection is the only way to reduce the frequency of complications and operative mortality [15].

Age, as well as the number of metastatic sites, should be taken into account when palliative resection is considered. Palliative resection may be beneficial for patients under 70 years of age if the tumor load is restricted to one metastatic site. Patients with low risk should undergo resection, and those with high risk should be selected for systemic chemotherapy or chemoradiation as required. This issue remains a matter of strong debate $[16,17]$.

In the present study, patients in the gastrojejunostomy group had low surgical morbidity and mortality, and we believe this procedure is an excellent way to reestablish the continuity of the alimentary tract. At present, the most controversial issue is the method of pyloric-obstruction management. A comparative study of traditional open gastrojejunostomy, laparoscopic gastrojejunostomy, and endoscopic pyloric stenting in malignant-obstruction management has been reported [18]; metal stents showed advantages over other stents, but this study did not consider a cost-benefit analysis [18]. To date, there has been no published randomized controlled trial comparing these methods; therefore, selection of laparoscopic gastrojejunostomy or endoscopic stenting should be included in the setting of clinical trials.

In our study, two patients in the $\mathrm{R} 2$ group had ascites and scarce peritoneal metastases, and surgical mortality can be associated with this condition. Some authors do not recommend palliative gastrectomy in patients with ascites [16].

The finding of distant metastases was not associated significantly with the appearance of complications in the present study. Nonetheless, some surgical groups do not perform palliative total gastrectomy in patients with inseparable attachments of GC to the posterior abdominal wall, tumor implants in the mesentery of the large bowel, liver metastases greater than an estimated onethird of liver mass, or a combination of the these factors [15]. However, the basis for these recommendations has not been clearly stated. The presence of single versus multiple metastatic sites or the presence of visceral versus transcoelomic metastasis were described as factors associated with higher surgical morbidity after total gastrectomy $[13,14]$.

Another observation that should be emphasized is survival. Although gastrojejunostomy is a procedure with less morbidity than $\mathrm{R} 1$ or $\mathrm{R} 2$ resection, overall survival in this group in our study was shorter. However, in this study, the follow up was poor because many patients were lost. Notwithstanding this, some authors agree that overall survival is not increased by palliative resection, even in the absence of randomized controlled trials on the subject.

Survival is an inadequate endpoint for evaluating the results of these palliative procedures. Quality of life and surgical morbidity and mortality should be the major outcome measurements in these studies, and survival should be secondary [19]. Therefore, a potential pitfall of our study is the absence of quality of life evaluations, explained by the retrospective nature of this series. To our knowledge, there is no randomized clinical trial that compares palliative chemotherapy with palliative resections, evaluating quality of life and surgical morbidity as endpoints.

In countries with a high frequency of patients with far-advanced disease, such as Mexico, where almost $70 \%$ of the patients must undergo palliative treatments only [3], it is a fundamental issue to define the role of palliative gastrectomy in the era of multimodal treatments.

In the near future, clinical trials must be directed to answer these questions.

\section{Conclusion}

The results of palliative gastrojejunostomy in our cohort were good and had acceptable morbidity and mortality. In patients with gross metastatic disease, potential benefits and risks must be balanced before palliative resection is undertaken, because surgical morbidity and mortality in this subgroup is high.

Acknowledgments Many thanks to Maria Esther Briones-Trejo and to Blanca Rosas-Rosas for their kind help with logistics, as well as to Isabel Sierra-Colindres and all personnel at the Clinical Archives. In addition, many thanks to Alejandra García-Hubard for her support in the writing of the manuscript and to Maggie Brunner, M. A., for her English-language editorial review.

\section{References}

1. Dicken BJ, Bigam DL, Cass C, Mackey JR, Joy AA, Hamilton SM. Gastric adenocarcinoma: review and considerations for future directions. Ann Surg 2005;241:27-39.

2. Munson JL, O'Mahony R. Radical gastrectomy for cancer of the stomach. Surg Clin North Am 2005;85:1021-32.

3. Oñate-Ocaña LF, Aiello-Crocifoglio V, Mondragón-Sánchez R, Ruiz Molina JM, Gallardo-Rincón D. Prognostic factors in 793 
cases of gastric cancer in an oncologic referral center. Rev Gastroenterol Mex 1999;64:114-21.

4. Miner TJ, Jaques DP, Karpeh MS, Brennan MF. Defining palliative surgery in patients receiving noncurative resections for gastric cancer. J Am Coll Surg 2004:198:1013-21.

5. McCahill LE, Smith DD, Borneman T, Juarez G, Cullinane C, Chu DZJ, et al. A prospective evaluation of palliative outcomes for surgery of advanced malignancies. Ann Surg Oncol 2003; 10:654-63.

6. Medina-Franco H, Contreras-Saldivar A, Ramos de la Medina A, Palacios-Sánchez P, Cortés-González R, Ugarte JA. Surgery for stage IV gastric cancer. Am J Surg 2004;187:543-6.

7. Wohrer SS, Raderer M, Hejna M. Palliative chemotherapy for advanced gastric cancer. Ann Oncol 2004;15:1585-95.

8. Greene FL, Page DL, Fleming ID. American Joint Committee on Cancer. AJCC Cancer staging manual, sixth edition. New York Berlin Heidelberg Tokyo: Springer-Verlag; 2002.

9. Oñate-Ocaña LF, Cortés-Cárdenas SA, Aiello-Crocifoglio V, Mondragón-Sánchez R, Ruiz-Molina JM. Preoperative multivariate prediction of morbidity after gastrectomy for adenocarcinoma. Ann Surg Oncol 2000;7:281-8.

10. Kaplan EL, Meier PM. Nonparametric estimation from incomplete observations. J Am Stat Soc 1958;53:457-81.

11. Bland JM, Altman DG. The logrank test. BMJ 2004;328:1073.

12. World Health Organization. Cancer pain relief and palliative care: report of a WHO Expert Committee. Geneva: World Health Organization;1990; 11: Technical report series no. 804.
13. Boodie AW, McMurtrey MJ, Giacco GG, McBride CM. Palliative total gastrectomy and esophagogastrectomy. A reevaluation. Cancer 1983;51:1195-200.

14. Lawrence W Jr, McNeer G. The effectiveness of surgery of palliation of incurable gastric cancer. Cancer 1958;11:28-32.

15. Saidi RF, ReMine SG, Dudrick PS, Hanna NN. Is there a role for palliative gastrectomy in patients with stage IV gastric cancer? World J Surg 2006;30:21-7.

16. Goel A, Khandelwal C. Role of palliative gastrectomy in locally advanced gastric cancer: experience of 313 cases. American Society of Clinical Oncology, American Society for Therapeutic Radiology and Oncology, American Gastroenterological Association and Society of Surgical Oncology: 2006 Gastrointestinal Cancers Symposium. www.asco.org (accessed December 6, 2006).

17. Hartgrink HH, Putter H, Klein Kranenbarg E, Bonenkamp JJ, van de Velde CJ; Dutch Gastric Cancer Group. Value of palliative resection in gastric cancer. Br J Surg 2002;89:1438-43.

18. Mittal A, Windsor J, Woodfield J, Casey P, Lane M. Matched study of three methods for palliation of malignant pyloroduodenal obstruction. Br J Surg 2004;91:205-9.

19. Blazeby JM, Avery K, Sprangers M, Pikhart H, Fayers P, Donovan J. Health-related quality of life measurement in randomized clinical trials in surgical oncology. J Clin Oncol 2006;24:3178-86. 